\title{
Analysis and Verification of Rotation Modulation Effects on Inertial Navigation System based on MEMS Sensors
}

\author{
Xueyun Wang, Jie $\mathrm{Wu}$, Tao Xu and Wei Wang \\ (School of Instrument Science and Opto-eletronics Engineering, Beihang University, \\ Beijing, China) \\ (E-mail: wangxueyun.buaa@gmail.com)
}

\begin{abstract}
Inertial Navigation Systems (INS) were large, heavy and expensive until the development of cost-effective inertial sensors constructed with Micro-electro-mechanical systems (MEMS). However, the large errors and poor error repeatability of MEMS sensors make them inadequate for application in many situations even with frequent calibration. To solve this problem, a systematic error auto-compensation method, Rotation Modulation (RM) is introduced and detailed. RM does no damage to autonomy, which is one of the most important characteristics of an INS. In this paper, the RM effects on navigation performance are analysed and different forms of rotation schemes are discussed. A MEMS-based INS with the RM technique applied is developed and specific calibrations related to rotation are investigated. Experiments on the developed system are conducted and results verify that RM can significantly improve navigation performance of MEMS-based INS. The attitude accuracy is improved by a factor of 5 , and velocity/position accuracy by a factor of 10 .
\end{abstract}

\section{KEY WORDS}

$\begin{array}{lll}\text { 1. Rotation Modulation. } & \text { 2. INS. } & \text { 3. MEMS Sensors. } \quad \text { 4alibration. }\end{array}$

Submitted: 4 December 2012. Accepted: 14 April 2013. First published online: 21 June 2013.

INTRODUCTION. Inertial navigation is a self-contained navigation technique in which measurements provided by accelerometers and gyroscopes are used to track the position and orientation of an object relative to a known starting point, orientation and velocity (Oliver, 2007). Inertial navigation is highly valued and widely used in many areas due to qualities such as autonomy, continuous navigation outputs and high accuracy over a short time. The most important part of an Inertial Navigation System (INS) is the Inertial Measurement Unit (IMU), which consists of three orthogonal accelerometers and three orthogonal gyroscopes. As the position and orientation of the vehicle are the integration results of accelerometers and gyroscopes respectively, the accuracy of INS is highly dependent on sensor errors, which include 
noise, bias, scale factor accuracy, temperature effects, etc. Micro-electro-mechanical system (MEMS) inertial sensors are a developing area, with promising advantages such as small size, low weight, rugged construction and short start-up time. Inexpensive to produce in high volume, they are compatible with operation in hostile environments. The current popularity of MEMS sensors in navigation systems has been well illustrated (Crain et al., 2010) and investigations of orientation estimation with MEMS sensors conducted (Lai et al., 2010). However, major disadvantages, which limit MEMS inertial sensors from a wider range of applications, are large errors. Moreover, some significant errors such as switch on/off bias are difficult to model and calibrate in normal ways. These are major bottlenecks for the further development of MEMS-based INS. To solve this problem and at the same time keep one of the most important features of INS, namely autonomy, a systematic error autocompensation method, Rotation Modulation (RM) is analysed, applied and verified in this paper.

Rotation Modulation is a technique where constant biases and slowly changing errors are modulated into zero-mean periodical values by rotating the inertial sensors around certain fixed axis (or axes) relative to the vehicle. Some research on RM technique was carried out in the past. In the 1950s, research was conducted to reduce gyroscope drifts by forcing the gimbals to rotate around one axis. Klass (1958) reported that Sperry Inc. adopted RM technique to eliminate the random biases of non-floated gyroscopes, cutting the random bias from $2-3 \%$ h to $0 \cdot 25^{\circ} / \mathrm{h}$. Giovanni and Levison (1981) presented a ring laser strap-down marine gyrocompass, which included the use of an indexer assembly to periodically rotate the inertial sensor cluster among four orthogonal dwell positions in the deck plane and this led to an associated significant improvement in heading accuracy. In 1987, Sperry Marine, Inc. and Honeywell Inc jointly developed a cost-effective high performance marine ring laser inertial navigator (MARLIN). This INS employed several accuracy enhancement techniques, including two-axis rotation (Levinson and Majure, 1987). Ishibashi and Aoki (2006) reported experiment results of an INS that was designed for Autonomous Underwater Vehicles (AUV) and applied the RM technique. It also verified that RM was an effective systematic scheme to improve navigation accuracy. Research of RM applied to INS or Attitude Heading Reference System (AHRS) based on Fibre-Optic Gyroscopes (FOG) has been reported recently (Sun et al., 2009, Wang and Xu, 2007).

According to the references mentioned above, all the INSs applied with RM technique are based on higher-grade inertial sensors, such as mechanical gyroscopes, Ring Laser Gyroscopes (RLG) or FOGs with mechanical or solid-state accelerometers. Little research has been done with the RM technique applied to an INS based on MEMS sensors. As high-performance navigation is well developed, the challenge now is to develop high-performance navigation solutions using low-cost sensor technology such as MEMS (Brown and Lu, 2004). In fact, due to MEMS sensor error characteristics, namely large bias and poor bias repeatability, RM can have excellent effects on MEMS-based INS. With the fast spread of MEMS sensors, the combination of RM and MEMS could possibly lead INS to have more application in areas such as guided bombs, commercial land vehicles etc. MEMS sensors are quite different from higher-grade sensors in many aspects, so a suitable rotation scheme has to be redesigned.

In this paper, the effects of RM on navigation performance are analysed in detail. Different rotation schemes are compared and a suitable one chosen for 
MEMS-based INS. The scheme, a Rotation Modulated Strap-down Inertial Navigation System (RMSINS), is developed, and the experiment results are presented to show that RM is an effective systematic error auto-compensation method to improve the navigation accuracy of Strap-down Inertial Navigation System (SINS) based on MEMS sensors.

\section{MODULATION EFFECTS ANALYSIS}

2.1. MEMS Sensor Error Characteristics. MEMS sensors' major errors consist of constant bias, thermo-mechanical white noise, flicker noise (bias instability), temperature effects and calibration errors (scale factor, linearity). For both MEMS gyroscopes and accelerometers, white noise errors (Angle/Velocity Random Walk) and uncorrected biases either due to uncompensated temperature fluctuations or an error in the initial bias estimation are usually the most important sources of error (Oliver 2007). Also, the repeatability of these errors, especially bias, is usually poor because of the environmental factor dependence (Aggarwal et al., 2007, El-Diasty et al., 2007). Even for MEMS-based INS and Global Positioning System (GPS) integrated navigation systems, the bias, which could possibly break up the Kalman Filter, is still the dominant error (Aggarwal et al., 2008). In conclusion, compensation for sensor bias is essential for a MEMS-based INS. Rotation Modulation can improve navigation performance by turning sensor constant bias and slowly-changing errors which cause inertial navigation errors growing with time into zero-mean periodical values which only result in limited errors. However, RM has no effect on the white noise that is a fast-changing error source. Wavelet decomposition can be applied to remove the high frequency noise that affects the sensors to obtain true angular rates and accelerations (Qian et al., 2010). Noise characteristics and stochastic model parameters can be determined to reduce the influence of high frequency noise (El-Diasty et al., 2006). For slowly changing errors that can be regarded as constants during one rotation cycle, the effects of RM are still accessible.

2.2. Coordinate Systems. There are three important coordinate systems for Rotation Modulated SINS: rotation coordinate system, body coordinate system and navigation coordinate system.

The rotation coordinate system is associated with the IMU. Its origin is the centre of the vehicle and axes point towards the sensitivity directions of the sensor triads assuming all the sensitivity directions are orthogonal.

The body coordinate system is associated with the vehicle. The origin is also the centre of the vehicle while $\mathrm{OX}_{\mathrm{b}}, \mathrm{OY}_{\mathrm{b}}$ and $\mathrm{OZ}_{\mathrm{b}}$ are aligned with pitch axis (right), roll axis (forward) and azimuth axis (up) respectively. The relationship between rotation coordinates and body coordinates is shown in Figure 1.

The navigation coordinate system is chosen as the Geography Frame which is defined by the rule that origin is the projection of the body coordinate system origin onto the Earth's geoid and $\mathrm{OX}_{\mathrm{n}}$ points East, $\mathrm{OY}_{\mathrm{n}}$ aligns North and $\mathrm{OZ}_{\mathrm{n}}$ coincides with Up.

2.3. North Channel Error Analysis. In previous research (Yang et al., 2005, Qian et al., 2010, Sun and Wang, 2012), the RM effects on sensor constant biases were detailed in formulas and expressions, but not for the effects on navigation performance. Hence the effects RM has on navigation error propagation are discussed here. Only the North Channel error is discussed because if errors in three-dimensional 


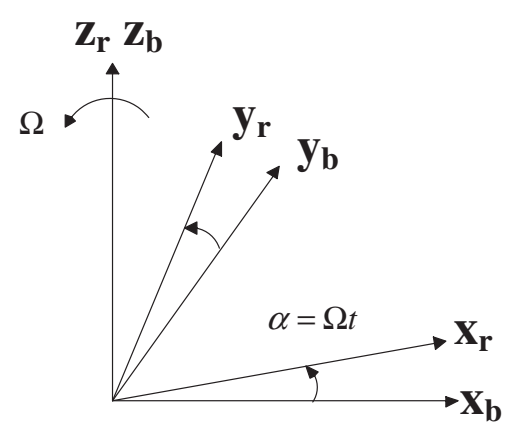

Figure 1. The Relationship Between Rotation Coordinates and Body Coordinates.

space are all involved, the analytical expressions will be extremely complicated, or even unachievable. The RM effects on sensor constant biases are briefly reviewed and then the effects on navigation error caused by gyroscope and accelerometer bias are detailed respectively through solving the inertial navigation equation set with the Laplace Transformation Method.

Assume the IMU is rotated at rate $\Omega$ around $\mathrm{OZ}_{\mathrm{b}}$. The rotation coordinate system coincides with the body coordinate system at the beginning. After time $t$, the angle from $\mathrm{OX}_{\mathrm{b}}$ to $\mathrm{OX}_{\mathrm{r}}\left(\mathrm{OY}_{\mathrm{b}}\right.$ to $\left.\mathrm{OY}_{\mathrm{r}}\right)$ is $\alpha=\Omega t$, so the transfer matrix from body coordinate system to rotation coordinate system is:

$$
C_{b}^{r}=\left[\begin{array}{ccc}
\cos \Omega t & \sin \Omega t & 0 \\
-\sin \Omega t & \cos \Omega t & 0 \\
0 & 0 & 1
\end{array}\right]=\left(C_{r}^{b}\right)^{T}
$$

Thus the inertial measurements can be expressed in body coordinate system as:

$$
\begin{aligned}
& \omega^{b}=C_{r}^{b} \omega^{r}+\left[\begin{array}{lll}
0 & 0 & -\Omega
\end{array}\right]^{\mathrm{T}} \\
& f^{b}=C_{r}^{b} f^{r}
\end{aligned}
$$

where $\omega^{r}$ and $f^{r}$ represent the inertial measurements in rotation coordinate system, namely the outputs of gyroscopes and accelerometers while $\omega^{b}$ and $f^{b}$ are the inertial measurements in body coordinate system which will be used to calculate the navigation results including attitude, velocity and position.

Assuming $\omega_{T}^{r}$ is the gyroscope output without errors and $\varepsilon_{x}, \varepsilon_{y}$ and $\varepsilon_{z}$ are the constant errors of three gyroscopes. Similarly $f_{T}^{r}$ is the accelerometer output without errors and $\nabla_{x}, \nabla_{y}$ and $\nabla_{z}$ are the constant errors of three accelerometers. Then the outputs of gyroscopes and accelerometers can be expressed as:

$$
\begin{aligned}
\omega^{r} & =\omega_{T}^{r}+\left[\begin{array}{lll}
\varepsilon_{x} & \varepsilon_{y} & \varepsilon_{z}
\end{array}\right]^{\mathrm{T}}+\left[\begin{array}{lll}
0 & 0 & \Omega
\end{array}\right]^{\mathrm{T}} \\
f^{r} & =f_{T}^{r}+\left[\begin{array}{lll}
\nabla_{x} & \nabla_{y} & \nabla_{z}
\end{array}\right]^{\mathrm{T}}
\end{aligned}
$$

Therefore, the angular rate and specific force in body coordinate system are:

$$
\omega^{b}=\omega_{T}^{b}+\left[\begin{array}{c}
\varepsilon_{x} \cos (\Omega t)-\varepsilon_{y} \sin (\Omega t) \\
\varepsilon_{y} \cos (\Omega t)+\varepsilon_{x} \sin (\Omega t) \\
\varepsilon_{z}
\end{array}\right], f^{b}=f_{T}^{b}+\left[\begin{array}{c}
\nabla_{x} \cos (\Omega t)-\nabla_{y} \sin (\Omega t) \\
\nabla_{y} \cos (\Omega t)+\nabla_{x} \sin (\Omega t) \\
\nabla_{z}
\end{array}\right]
$$


Clearly the constant biases of both gyroscopes and accelerometers along $\mathrm{OX}_{\mathrm{r}}$ and $\mathrm{OY}_{\mathrm{r}}$ are turned into zero-mean periodical values in the body coordinate system but everything along $\mathrm{OZ}_{\mathrm{b}}$ remains the same. In conclusion, only the constant errors along the axes that are perpendicular to the rotation axis can be modulated. When these modulated errors are involved in the inertial navigation error propagation, the RM effects on navigation accuracy can be observed and analysed. Take the North Channel as an example. The simplified velocity/position error model for the North Channel can be expressed in terms of the following set of coupled differential equations (Titterton and Weston, 2004):

$$
\begin{aligned}
& \delta \dot{\beta}=-\left(\omega_{i e} \cos L+v_{E} / R\right) \delta \gamma-\delta v_{N} / R+\varepsilon_{x} \\
& \delta \dot{\gamma}=\varepsilon_{z} \\
& \delta \dot{v}_{N}=g \delta \beta+\nabla_{y} \\
& \delta \dot{x}_{N}=\delta v_{N}
\end{aligned}
$$

where $\varepsilon_{x}$ and $\varepsilon_{z}$ are gyroscope biases along the East and Up respectively; $\nabla_{y}$ is the bias of the north accelerometer and $R$ is the radius of the Earth. $\omega_{i e}$ is the Earth rotation rate and $L$ is the local longitude. $\delta \gamma$ and $\delta \beta$ are the azimuth and pitch error respectively while $\delta v_{N}$ and $\delta x_{N}$ are the north velocity and position errors respectively.

Firstly, taking just the east gyroscope constant bias into account, all other errors are set to zero. With RM, constant bias $\varepsilon_{x}$ in the rotation coordinate system is turned into $\varepsilon_{x} \cos (\Omega t)$ in body coordinate system and the differential equations are presented as:

$$
\begin{aligned}
& \delta \dot{\beta}=-\delta v_{N R} / R+\varepsilon_{x} \cos (\Omega t) \\
& \delta \dot{v}_{N R}=g \delta \beta \\
& \delta \dot{x}_{N R}=\delta v_{N R}
\end{aligned}
$$

With the Laplace Transformation Method, the differential equations are turned into normal equations:

$$
\begin{aligned}
& s \delta \beta(s)=-\delta v_{N R}(s) / R+\varepsilon_{x} \frac{s}{s^{2}+\Omega^{2}} \\
& s \delta v_{N R}(s)=g \delta \beta(s) \\
& s \delta x_{N R}(s)=\delta v_{N R}(s)
\end{aligned}
$$

Being solved and applied with Laplace Inverse Transformation, $\delta v_{N}$ and $\delta x_{N}$ are expressed in time domain in the following forms:

$$
\begin{aligned}
& \delta v_{N R}=\frac{g}{\Omega_{s}^{2}-\Omega^{2}}\left[\cos (\Omega t)-\cos \left(\Omega_{s} t\right)\right] . \varepsilon_{x} \approx \frac{g[1-\cos (\Omega t)]}{\Omega^{2}} \varepsilon_{x} \\
& \delta x_{N R}=\frac{g}{\Omega_{s}^{2}-\Omega^{2}}\left[\frac{\sin (\Omega t)}{\Omega}-\frac{\sin \left(\Omega_{s} t\right)}{\Omega_{s}}\right] . \varepsilon_{x} \approx \frac{g}{\Omega^{2}}\left[t-\frac{\sin (\Omega t)}{\Omega}\right] \varepsilon_{x}
\end{aligned}
$$

where $\Omega_{s}=\sqrt{g / R}$ is the Schuler Radian Frequency. The approximate condition is that operation time is very short compared with the Schuler Period (84.4 minutes) and rotation rate is much larger than Schuler Radian Frequency $(0 \cdot 00124 \mathrm{rad} / \mathrm{s})$. 


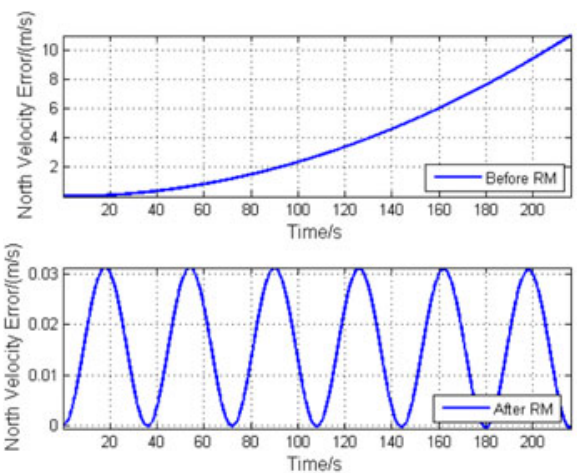

(a)Velocity Error Before and After RM
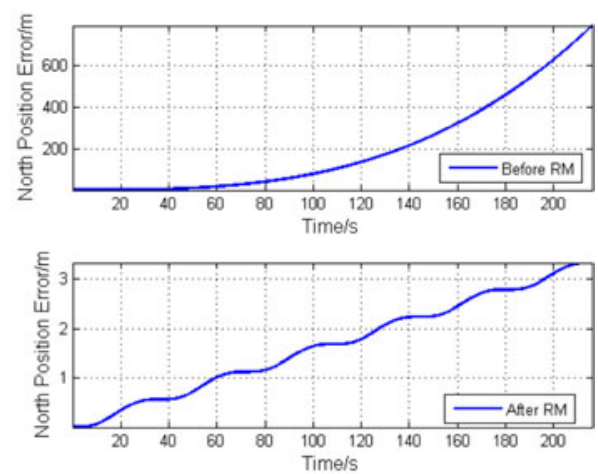

(b) Position Error Before and After RM

Figure 2. Simulation of North Velocity/Position Error Caused by East Gyroscope Bias.

With the same algorithm, the velocity and position errors before RM can be solved and expressed as:

$$
\begin{aligned}
& \delta v_{N}=R\left[1-\cos \left(\Omega_{s} t\right)\right] \varepsilon_{x} \approx \frac{1}{2} g \varepsilon_{x} \cdot t^{2} \\
& \delta x_{N}=R\left[t-\sin \left(\Omega_{s} t\right) / \Omega_{s}\right] \varepsilon_{x} \approx \frac{1}{6} g \varepsilon_{x} \cdot t^{3}
\end{aligned}
$$

Figure 2 shows the navigation error comparison between after and before RM. Assume $\varepsilon_{x}$ is $10 \%$ h and rotation rate $\Omega$ is $10 \%$ s. Clearly the velocity/position accuracy is greatly improved because the error caused by east gyroscope bias is significantly constrained by RM. For short time inertial navigation, the position error grows with respect to time at rate $g \varepsilon_{x} / \Omega^{2}$ after RM while it increases with the cubic of operation time at rate $g_{\varepsilon_{x}} / 6$ before RM.

Secondly, taking just the north accelerometer constant bias into account, all other errors are set to zero. The error equation set turns into another form:

$$
\begin{aligned}
& \delta \dot{\beta}=-\delta v_{N} / R \\
& \delta \dot{v}_{N}=g \delta \beta+\nabla_{y} \\
& \delta \dot{x}_{N}=\delta v_{N}
\end{aligned}
$$

Following the same procedure conducted above, the velocity and position error after and before RM can be obtained:

$$
\begin{gathered}
\delta v_{N R}=\frac{\nabla_{y}}{\Omega_{s}^{2}-\Omega^{2}}\left[\Omega_{s} \sin \left(\Omega_{s} t\right)-\Omega \sin (\Omega t)\right] \approx \frac{\sin (\Omega t)}{\Omega} \nabla_{y} \\
\delta x_{N R}=\frac{\nabla_{y}}{\Omega_{s}^{2}-\Omega^{2}}\left[\cos (\Omega t)-\cos \left(\Omega_{s} t\right)\right] \approx \frac{1-\cos (\Omega t)}{\Omega^{2}} \nabla_{y} \\
\delta v_{N}=\frac{\nabla_{y}}{\Omega_{s}} \sin \left(\Omega_{s} t\right) \approx \nabla_{y} t \\
\delta x_{N}=\frac{\nabla_{y}}{\Omega_{s}^{2}}\left[1-\cos \left(\Omega_{s} t\right)\right] \approx 0 \cdot 5 \nabla_{y} t^{2}
\end{gathered}
$$




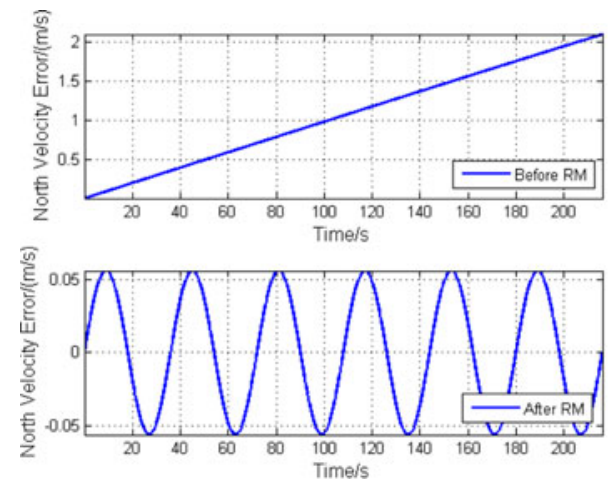

(a)Velocity Error Before and After RM
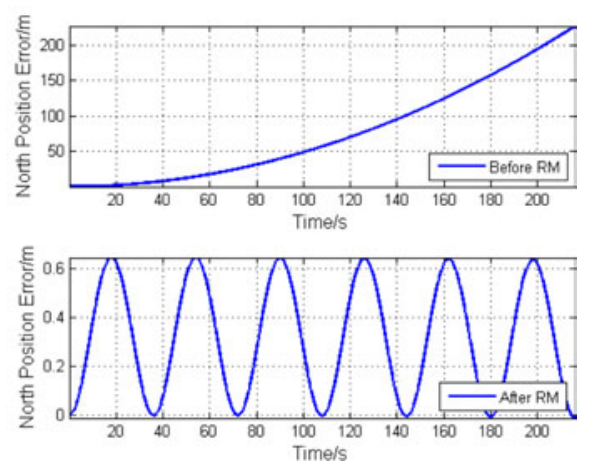

(b) Position Error Before and After RM

Figure 3. Simulation of Velocity/Position Error Caused by North Accelerometer Bias.

Figure 3 shows the navigation error comparison before and after RM. Assume $\nabla_{y}$ is $1 \mathrm{mg}$ and rotation rate $\Omega$ is $10 \%$. Similarly RM turns velocity/position errors caused by accelerometer bias into periodical values instead of divergent errors, so RM can also reduce the navigation error caused by accelerometer bias.

We can see that the navigation errors are related to the rotation rate $\Omega$. In most cases $\Omega$ is much higher than $\Omega_{s}$, so the higher $\Omega$ is, the smaller navigation errors are. In practice, higher $\Omega$ may cause problems that may degrade the performance of RM. For instance, when $\Omega$ is high, it becomes harder to keep rotation steady. Rotation instability may cause gyroscope outputs with serious high-frequency noise, which cannot be modulated by RM. Besides, higher rotation rates demand more specific calibrations related to rotation. The rotation rate has to be determined according to specific sensors, systems and applications. This necessitates a balance between modulation effects and control difficulty. Generally speaking, for high accuracy sensors, the rotation rate should be low (e.g. $1 \%$ s) to avoid too much distortion being introduced by rotation. As the uncorrected biases of high accuracy sensors are small, the rotation rate doesn't have to be high. However, for low accuracy sensors like MEMS sensors, the rotation rate has to be much higher, e.g. $10 \%$ s to $30 \%$, to achieve better RM effects on navigation accuracy. With an even higher rotation rate, the difficulty in controlling the motor increases greatly. Poor control characteristics will directly result in poor navigation accuracy, so we must normally avoid setting the rotation rate too high.

3. ROTATION SCHEME COMPARISIONS. As MEMS sensor error characteristics are quite different from those of higher-grade inertial sensors, a suitable rotation scheme that is discriminative from previous solutions must be designed properly for RMSINS based on MEMS sensors. We start by comparing various rotation schemes. There are several ways to sort rotation schemes according to different criteria, such as the number of rotation axes, direction of rotation, continuity of rotation etc. Advantages and defects of each rotation scheme are examined. 


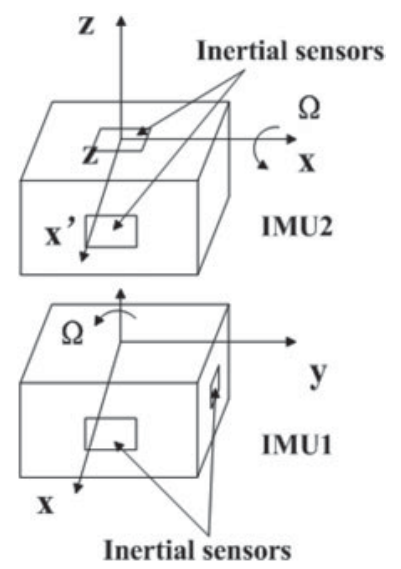

Figure 4. Dual-axis Rotation Scheme (2 IMUs).

\subsection{Number of Rotation Axes}

3.1.1. Single Axis. The most obvious defect of single-axis rotation is that the constant errors along the rotation axis cannot be modulated and gyroscope outputs may get worse if rotation distortions are involved. However, advantages including simple structure, small size, low cost and high robustness are obvious. If a better gyroscope is applied on the rotation axis, or the performance requirement along the rotation axis is lower than other axes, as in the cases of missiles and guided bombs whose accuracy demand for roll is much lower than that of azimuth and pitch, singleaxis rotation is a better choice. Due to the simple algorithm and easy implementation, much research has been conducted on single-axis rotation (Yang et al., 2005, Sun et al., 2012).

3.1.2. Multiple Axes. Rotation schemes with more than one rotation axis guarantee that constant errors along every direction are modulated; futhermore, one (for double-axis rotation) or three (for triple-axis rotation) redundant sensor pairs are available, which increases the reliability of INS. However, a much more complex, higher cost and larger volume structure results. Figure 4 is a block diagram of a dualaxis rotation scheme. The implementation and capacities of an INS with double-axis rotation were described and analysed (Li et al., 2010).

Generally, multiple-axis-rotation schemes are adopted for high accuracy gyroscopes and accelerometers in applications with long time/distance requirements, (e.g. ships/submarines) because all constant errors must be compensated to provide three-dimensional high-accuracy performance. Single-axis rotation is a better solution for low accuracy, low cost, small size INS applied for short time/distance navigation (e.g. missiles/guided bombs). Only by reducing the cost, weight, volume and complexity of IMU as much as possible can MEMS sensors' advantages still benefit INS, so a single-axis-rotation scheme is the better choice for RMSINS based on MEMS sensors.

\subsection{Direction of Rotation}

3.2.1. Unidirectional Rotation. The IMU is rotated around one direction as shown in Figure 5(a). In this case, a slip ring which can deliver signals between a rotating part and a static one is generally used to send the raw data from all sensors 
to CPUs for calibration and navigation computation. This increases cost, and the reliability of a slip ring is usually much lower than that of MEMS sensors, weakening the advantage of high reliability. The superiority of unidirectional rotation is easier motor control with no direction changes.

3.2.2. Reciprocating Rotation. Rotating direction is changed when the IMU covers a certain angle, e.g. $360^{\circ}$ or $720^{\circ}$, as shown in Figure 5(b). In this case, slip rings may be replaced with ordinary wires, reducing cost and improving reliability. Reciprocating rotation is also better at restraining constant errors and results in better performance, assuming all other conditions are identical. The supporting experiments were conducted but reasons were not discussed or provided (Ishibashi and Aoki, 2006). Explanations are as follows:

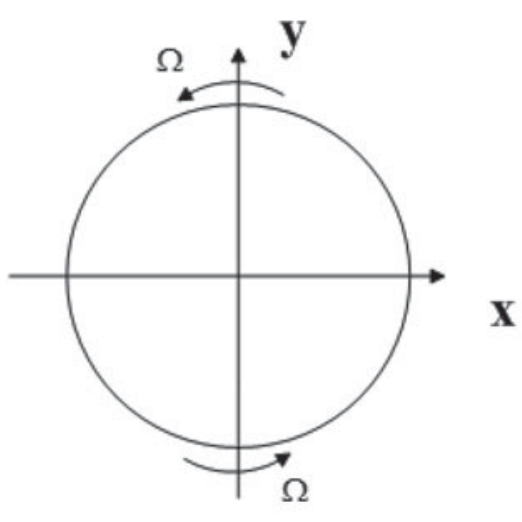

(a) Unidirectional Rotation Scheme

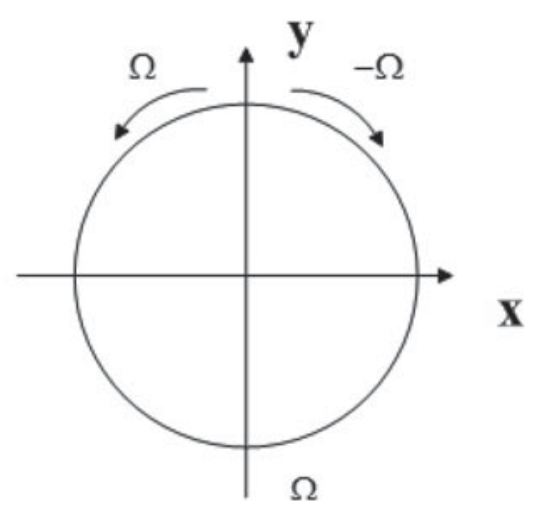

(b) Reciprocating Rotation Scheme

Figure 5. Rotation Schemes With Different Rotating Direction.

Assume that the rotation axis is identical with $\mathrm{OZ}_{\mathrm{b}} . \varepsilon_{x}, \varepsilon_{y}$ are the gyroscope drifts along $\mathrm{OX}_{\mathrm{r}}$ and $\mathrm{OY}$ respectively. To simplify the algorithm, set $\varepsilon_{x}=\varepsilon_{y}$ and all other errors zero. The rotation rate is $\Omega$ and the body coordinate system is identical with the navigation coordinate system at the beginning. Thus the equivalent east gyroscope drift is:

$$
\varepsilon_{E}=\varepsilon_{x}(\cos \Omega t+\sin \Omega t)
$$

The pitch error and north velocity over a short operation time are respectively:

$$
\begin{aligned}
\Delta \phi_{E} & =\int_{0}^{t} \varepsilon_{E} \mathrm{~d} t=\frac{\varepsilon_{x}}{\Omega}(1-\cos \Omega t+\sin \Omega t) \\
\Delta V_{N} & =\int_{0}^{t} g \cdot \Delta \phi_{E} \mathrm{~d} t=\frac{g \varepsilon_{x}}{\Omega}\left[t-\frac{\sin \Omega t+\cos \Omega t+1}{\Omega}\right]
\end{aligned}
$$

For unidirectional rotation, if the time the IMU takes to cover $360^{\circ}$ is $T$, which means the rotation modulation period is $T$, then the average equivalent gyroscope drift is 0 in $2 T$, and the average pitch error is $\varepsilon_{x} / \Omega$ while the average north velocity error is divergent. For reciprocating rotation, however, as the RM period doubles to 
Table 1. The Average Errors in $2 T$ for Different Rotation Direction Schemes.

\begin{tabular}{lcc}
\hline & \multicolumn{2}{c}{ Average Error in $2 T$} \\
\cline { 2 - 3 } Error Sources & Unidirectional Rotation & Reciprocating Rotation \\
\hline$\varepsilon_{E}$ & 0 & 0 \\
$\Delta \phi_{E}$ & $\varepsilon_{x} / \Omega$ & 0 \\
$\Delta V_{N}$ & Diverges & $-\pi g \varepsilon_{x} / \Omega^{2}$ \\
\hline
\end{tabular}

$2 T$ and rotation rate $\Omega$ changes to $-\Omega$ after time $T$, not only the average equivalent gyroscope drift but also the average pitch error is 0 in $2 T$. As for the north velocity error, it no longer diverges and the average in $2 T$ is $-\pi g \varepsilon_{x} T / \Omega$. From Table 1, it is clear that reciprocating rotation has better modulation effects on navigation performance than unidirectional rotation, if other conditions are identical.

\subsection{Continuity of Rotation}

3.3.1. Discontinuous Rotation. IMU stops at certain positions, e.g. $0^{\circ}$ and $180^{\circ}$. During the rotation, IMU arrives at one specific position, stays there for some time and then goes to the next stop. Since the rotation time is usually much shorter than stop time, the equivalent sensor errors are characterized as a rectangular signal. Discontinuous rotation may be a suitable scheme for RM of high-accuracy INS because the distortion caused by rotation disappears when IMU stops. The rotation rate is usually low in discontinuous rotation schemes; control of the motor is more accurate.

3.3.2. Continuous Rotation. IMU keeps running without any stops or changes except for the turnovers. In this case, the equivalent inertial sensor errors are characterized as sinusoidal values. As MEMS sensors' noise and biases are high, the navigation errors will rise if IMU stops, so continuous rotation is a better choice for MEMS-based INS. Also, as IMU rotation rate increases, the stop/start motor control required for discontinuous rotation increases in difficulty, making such schemes unfeasible.

To summarise, a single-axis continuous reciprocating rotation scheme was chosen and applied to a SINS based on MEMS sensors, which is called Rotation Modulation Strap-down Inertial Navigation System (RMSINS). The rotation axis is identical with $\mathrm{OZ}_{\mathrm{b}}$ and the rotation rate is $10 \%$ s.

\section{IMPLEMENTATION OF RMSINS}

4.1. Hardware Structure. RMSINS consists of four parts: IMU, rotary components, electronics and the frame. The schematic diagram and the picture are shown in Figure 6.

The IMU is composed of one three axis gyroscope (STIM202, Sensonor) and three single axis accelerometers (MS8010, Colibrys). All the inertial sensors are based on MEMS technology. The gyroscope bias instability is $8.6 \%$ hy standard deviation; the Angle Random Walk is $1.83^{\circ} / \sqrt{\mathrm{h}}$ by Root Allen Variance and the scale factor accuracy is within $\pm 0.2 \%$. As for the accelerometers, the bias instability is $2.3 \mathrm{mg}$ by standard deviation; the Velocity Random Walk is $0.124 \mathrm{~m} / \mathrm{s} / \sqrt{\mathrm{h}}$ by Root Allen Variance and the scale factor accuracy is within $\pm 0.18 \%$. All the parameters are 


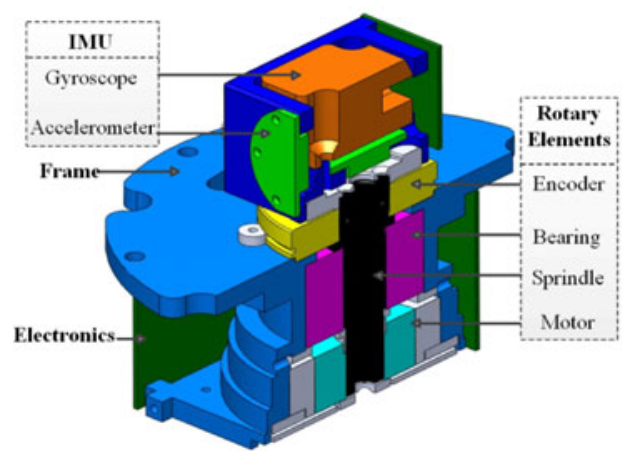

(a) Schematic Diagram

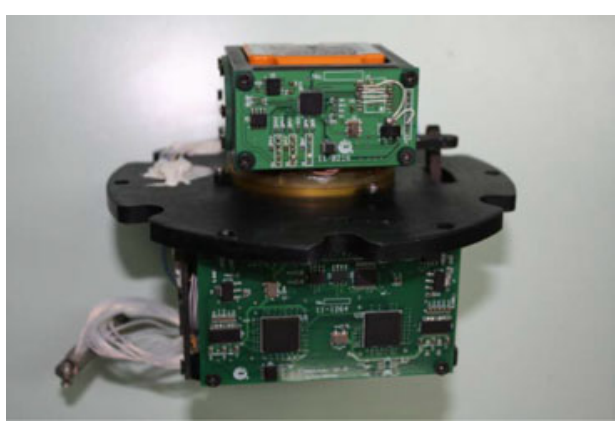

(b) Picture

Figure 6. Structure Pictures of RMSINS.

obtained through tests in laboratory conditions. As fixed biases and scale factors can be calibrated, only stochastic errors are described here. Benefitting from the MEMS inertial sensors, the IMU is very small $(40 \mathrm{~mm} \times 40 \mathrm{~mm} \times 20 \mathrm{~mm})$, rigid and with low power consumption (less than $1 \mathrm{~W}$ ). The IMU is rotated by a torque motor around $\mathrm{OZ}_{\mathrm{b}}$. Rotation rate along $\mathrm{OZ}_{\mathrm{b}}$ is sensed and it must be removed from the gyroscope outputs.

Rotary components refer to an optoelectronic encoder (DS58-20, Netzer), a torque motor, a bearing and a spindle. The encoder is an angle sensor that provides the exact angular position (its accuracy is within $2^{\prime \prime}$ ) relative to a starting point. The encoder is an essential component of RMSINS, providing the differential results from the angular positions that correct gyroscope outputs along $\mathrm{OZ}_{\mathrm{b}}$ and the angular position and velocity for motor control as feedbacks.

The electronics provide signal processing, motor control, navigation computation and data output etc. The CPU is a DSP (TMS320F28335) produced by TI Corp. With the aim of compensating for the time delay discrepancy among different sensors and improving the pre-processing, two DSPs are designed to complete all the system's functions. One is for the pre-processing of raw data from the inertial sensors and encoder, as well as controlling the motor, while the other implements the inertial navigation algorithm and system interface, sending navigation results and receiving initialization information and control orders. Initialization information comprises position, velocity and azimuth while control orders include setting motor rotating or static, changing the rotation rate etc. The electronics schematic is shown in Figure 7.

The frame supports all other parts and connects with the shell. There is a big plate in the middle of the frame, whose function is to separate the system into two chambers in the shell. The IMU is placed in one chamber with the aim of creating a stable temperature and magnetic field to reduce their influences on MEMS sensors. The size of the RMSINS is $\phi 95 \mathrm{~mm} \times 106 \mathrm{~mm}$ and its weight is less than 3 kilograms.

Overall, our RMSINS based on MEMS sensors is much smaller, lighter and cheaper than a SINS based on optical or mechanical inertial sensors, although added accessories may increase the expense and volume to some extent. However, RM will greatly enhance the performance of the INS, and significantly improve the price/ performance ratio of the system. 


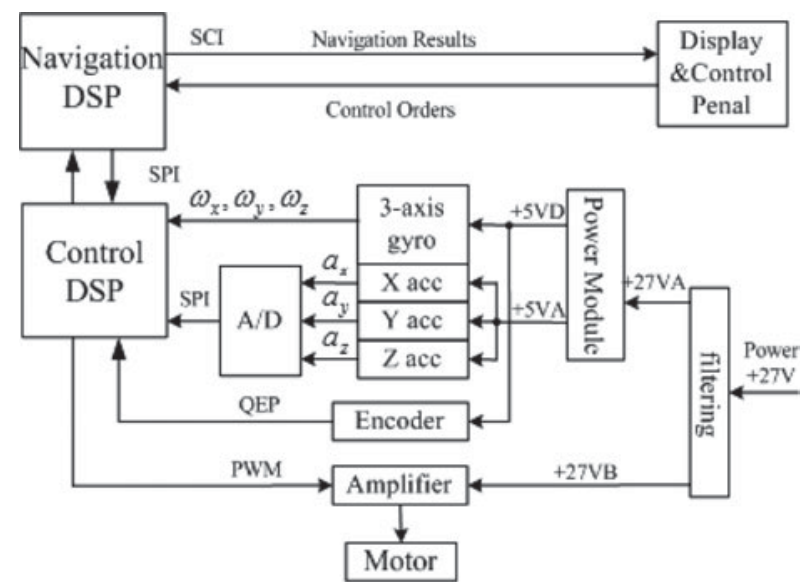

Figure 7. Electronics Schematic.

\subsection{Navigation Algorithm}

4.2.1. Initial Alignment. As the inertial navigation results are achieved by integral mathematics that needs an origin, initial alignment determines the origin, and must be finished before inertial navigation. 3-D attitudes, 3-D velocities and 3-D positions define the origin. However INS alone cannot achieve all nine parameters. Velocity and position are given by other facilities, for example Global Positioning System (GPS). INS itself can obtain the 3-D attitudes, but the goals of initial alignment vary depending on the type of INS. For platform inertial navigation systems (PINS), the goal of initial alignment is to set the platform frame coordinate with the navigation frame; initial attitudes are then given by the angle sensors on gimbals. For strap-down inertial navigation systems (SINS), initial alignment estimates the original 3-D attitudes through the outputs of inertial sensors.

Theoretically, INS can achieve all 3-D attitudes autonomously using gravity and the rotation rate of the Earth. Pitch and roll accuracy is determined by accelerometer biases while azimuth accuracy is dependent on gyroscope drifts. The MEMS accelerometers used in RMSINS are capable of initializing pitch and roll with accuracy less than $0.05^{\circ}$ with the help of RM. The algorithm is shown below.

$$
\begin{aligned}
& \theta_{0}=\sin ^{-1}\left(\overline{a_{y}^{b}} / g\right) \\
& \gamma_{0}=\sin ^{-1}\left(-\overline{a_{x}^{b}} / g\right)
\end{aligned}
$$

where $\theta_{0}$ is the initial pitch, $\gamma_{0}$ is the initial roll, $g$ is the local gravity and $\overline{a_{y}^{b}}, \overline{a_{x}^{b}}$ are the averages of specific force in body coordinate system, which can be obtained through transfer as follows:

$$
\begin{aligned}
& a_{x}^{b}=a_{x}^{r} \cos \alpha-a_{y}^{r} \sin \alpha \\
& a_{y}^{b}=a_{y}^{r} \cos \alpha+a_{x}^{r} \sin \alpha
\end{aligned}
$$

where $a_{x}^{r}$ and $a_{y}^{r}$ represent the outputs of accelerometers and $\alpha$ is the IMU's angular position which is given by the encoder.

However, low grade gyroscopes such as MEMS-based sensors suffer from bias instability and noise levels that can completely mask Earth's rotation rate, the 


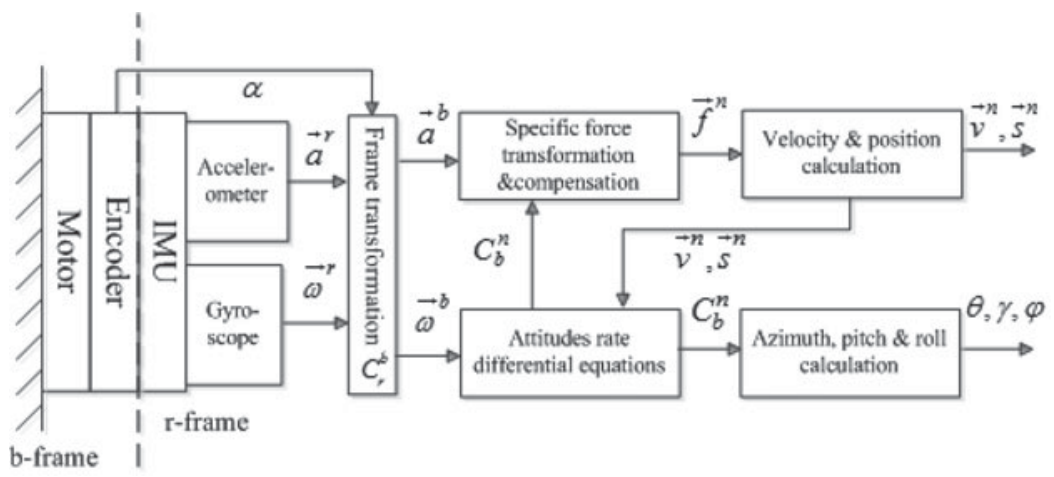

Figure 8. Navigation Algorithm Schematic.

reference signal for azimuth estimation, so it is not feasible to achieve initial azimuth by RMSINS alone. As a result, the initial azimuth, as well as position and velocity, is obtained from other facilities to finish the initial alignment of RMSINS. Position and velocity can be given to RMSINS by GPS and a Magnetic Heading System can give azimuth. All these parameters can be inputted through specific software on a laptop if necessary. The provided information is used directly by RMSINS as the initial status.

4.2.2. Inertial Navigation. Strap-down inertial navigation requires orientation, velocity and position updates. Detailed explanations of the conventional algorithm have been made (Titterton and Weston 2004). For RMSINS, the difference is the rotation of the IMU, which requires a transfer before the conventional algorithm can be applied.

As mentioned in Section 2.3, the transfer matrix from rotation coordinate system to body coordinate system is:

$$
C_{r}^{b}=\left[\begin{array}{ccc}
\cos \alpha & -\sin \alpha & 0 \\
\sin \alpha & \cos \alpha & 0 \\
0 & 0 & 1
\end{array}\right]
$$

So the angular velocity and specific force in body coordinate system can be expressed as:

$$
\begin{aligned}
{\left[\begin{array}{lll}
\omega_{x}^{b} & \omega_{y}^{b} & \omega_{z}^{b}
\end{array}\right]^{T}=C_{r}^{b}\left[\begin{array}{lll}
\omega_{x}^{r} & \omega_{y}^{r} & \omega_{z}^{r}
\end{array}\right]^{T}+\left[\begin{array}{lll}
0 & 0 & -\Omega
\end{array}\right]^{T} } \\
{\left[\begin{array}{lll}
a_{x}^{b} & a_{y}^{b} & a_{z}^{b}
\end{array}\right]^{T}=C_{r}^{b}\left[\begin{array}{lll}
a_{x}^{r} & a_{y}^{r} & a_{z}^{r}
\end{array}\right]^{T} }
\end{aligned}
$$

where $\left[\begin{array}{lll}a_{x}^{r} & a_{y}^{r} & a_{z}^{r}\end{array}\right]^{T}$ and $\left[\begin{array}{ccc}\omega_{x}^{r} & \omega_{y}^{r} & \omega_{z}^{r}\end{array}\right]^{T}$ are the outputs of accelerometers and gyroscopes respectively.

After obtaining the angular velocity and specific force in the body coordinate system, the conventional strap-down inertial navigation algorithm can be applied to RMSINS. The algorithm scheme is shown in Figure 8.

5. CALIBRATIONS. Calibrations for RMSINS are identical to those for ordinary SINS if the motor is locked, except for those related to rotation. A standard 


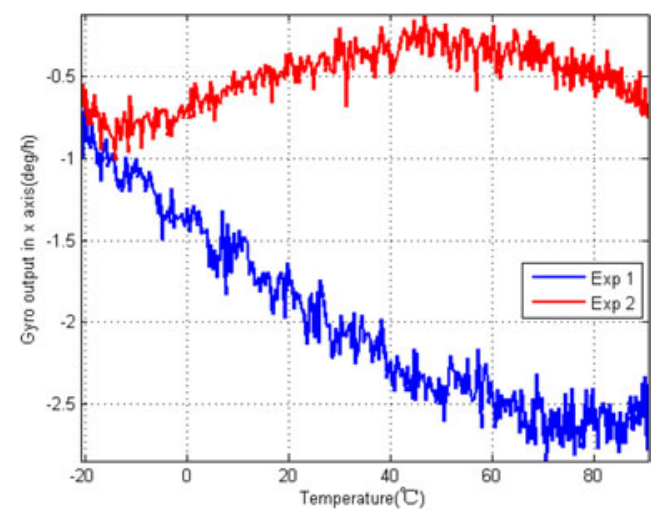

Figure 9. Poor Repeatability of Gyroscope Temperature Bias.

testing and calibration procedure for low cost MEMS inertial sensors and units was developed by researchers from University of Calgary (Aggarwal et al., 2008). It involves the thermal model, biases, scale factors and non-orthogonalities and provides practical ways to complete the standard calibration. Lai (2010) detailed similar calibration procedures which could also be used for conventional calibration of lowaccuracy MEMS inertial sensors triads.

In this system, the extended six-position calibration and angular rate tests are adopted to obtain the biases, scale factors and non-orthogonalities of the sensor triads. Thermal bias models for accelerometers are designed, but thermal bias models for gyroscopes are not developed for two reasons: firstly, thermal effect has been factory-calibrated, limiting the bias variation with temperature to an acceptable level; and secondly, the repeatability of gyroscope bias on temperature is so poor that it is not feasible to calibrate it, as shown in Figure 9. Temperature readings come from sensors inside the gyroscope. Experiments were conducted twice under similar conditions but gyroscope outputs were quite different, proving the repeatability of gyroscope temperature bias is very poor. However as the bias caused by temperature is slowly changing, it can be effectively modulated by rotation.

After normal calibration, an orthogonal coordinate system, identical to the body coordinate system, is established. Correct measurements can then be obtained and the strap-down inertial navigation algorithm can calculate the orientation, velocity and position.

However, after introducing Rotation Modulation, more calibrations are needed to guarantee that rotation does not incorporate distortions and errors. There are three main types of calibration: firstly, the calibration of non-orthogonality of rotation axis and body coordinate system, namely $\mathrm{OX}_{\mathrm{b}}$ and $\mathrm{OY}_{\mathrm{b}}$; secondly, calibration of centripetal acceleration caused by rotation and finally, calibration of the synchronism of gyroscope and encoder.

5.1. Calibration of Non-orthogonality of Rotation Axis and Body Coordinates. Defined as the direction of rotation rate, the rotation axis is determined by the motor and cannot be chosen. It is unlikely to align with the $\mathrm{OZ}_{\mathrm{b}}$ which is defined by the system structure due to assembly error. Without calibration, the rotation rate will project on $\mathrm{OX}_{\mathrm{b}}$ and $\mathrm{OY}_{\mathrm{b}}$ and these projections will cause serious problems as the 


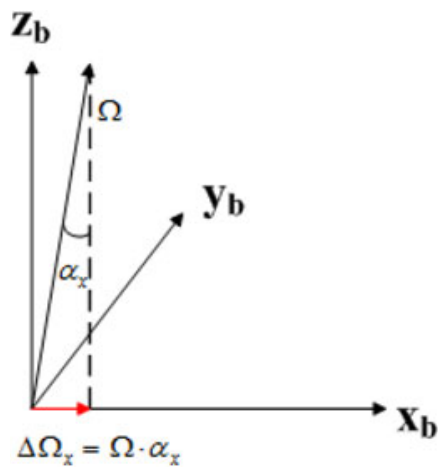

Figure 10. Error Caused by the Non-orthogonality of Rotation Axis and Body Coordinates.

rotation rate is quite high. Assuming the non-orthogonal angle between the rotation axis and $\mathrm{OX}_{\mathrm{b}}$ is $1^{\prime}$, and the rotation rate is $10 \%$, then the projection on $\mathrm{OX}_{\mathrm{b}}$ is:

$$
\Delta \Omega_{x}=\Omega \cdot \sin \alpha_{x} \approx \Omega \cdot \alpha_{x}=10.5 \mathrm{deg} / \mathrm{h}
$$

This error functions as the gyroscope constant bias as indicated in Figure 10 but cannot be modulated through rotation, as it is itself caused by rotation. Obviously, this will degrade the navigation accuracy of RMSINS, possibly to an extent where it is poorer than that of SINS without RM, so this non-orthogonality must be compensated. However, the small fraction of misalignment between $\mathrm{OZ}_{\mathrm{b}}$ and the rotation axis is of little influence as shown below assuming the misalignment angle is 1', too:

$$
\Delta \Omega_{z}=\Omega \cdot\left(1-\cos \alpha_{z}\right)=1.5 \times 10^{-3} \mathrm{deg} / \mathrm{h}
$$

Hence, calibrating the misalignment between $\mathrm{OZ}_{\mathrm{b}}$ and the rotation axis is unnecessary.

In order to calibrate the non-orthogonality of rotation axis and $\mathrm{OX}_{\mathrm{b}} / \mathrm{OY}_{\mathrm{b}}$, RMSINS is mounted on a levelled static platform. The calibration method for either axis is the same, so $\mathrm{OX}_{\mathrm{b}}$ is taken for example. With $\mathrm{OX}_{\mathrm{b}}$ pointing North, the IMU is rotated at rate $\Omega$ in one direction for a circle and then the same rate in the other direction for another circle. Assuming the non-orthogonal angle is $\alpha_{x}$ and the constant bias is $\varepsilon_{x}$ (in a short time such as several minutes, the gyroscope drifts can be regarded as constant biases), the gyroscope outputs in rotation coordinate system can be expressed as:

$$
\begin{aligned}
& \omega_{x 1}=\Omega \alpha_{x}+\varepsilon_{x}+\omega_{N} \cos (\Omega t) \\
& \omega_{x 2}=-\Omega \alpha_{x}+\varepsilon_{x}+\omega_{N} \cos (-\Omega t)
\end{aligned}
$$

where $\omega_{N}$ is the northern component of the rotation rate of the Earth. The relation between $\alpha_{x}$ and gyroscope outputs can be expressed as follows:

$$
\bar{\omega}_{x 1}-\bar{\omega}_{x 2}=2 \alpha_{x} \cdot \Omega
$$

where $\bar{\omega}_{x 1}$ and $\bar{\omega}_{x 2}$ are the average of the gyroscope outputs. This procedure should be repeated several times with different rotation rate $\Omega$. Applying Least Square method, the non-orthogonal angle between the rotation axis and $\mathrm{OX}_{\mathrm{b}}$ can be obtained. Results are shown in Table 2 and Fig. 11. The IMU was rotated at rate $10 \%$. The constant bias and the Earth rotation rate were removed from the raw data. 
Table 2. Calibration Results of Non-orthogonality of Rotation Axis and Body Coordinates.

\begin{tabular}{lccccccc}
\hline Items(") & 1 & 2 & 3 & 4 & 5 & Average & Deviation \\
\hline$\alpha_{x}$ & $244 \cdot 4$ & $244 \cdot 5$ & $244 \cdot 3$ & $244 \cdot 2$ & $244 \cdot 8$ & $244 \cdot 44$ & $0 \cdot 23$ \\
$\alpha_{y}$ & $238 \cdot 2$ & $238 \cdot 5$ & $238 \cdot 5$ & $238 \cdot 6$ & $238 \cdot 8$ & $238 \cdot 52$ & $0 \cdot 22$ \\
\hline
\end{tabular}

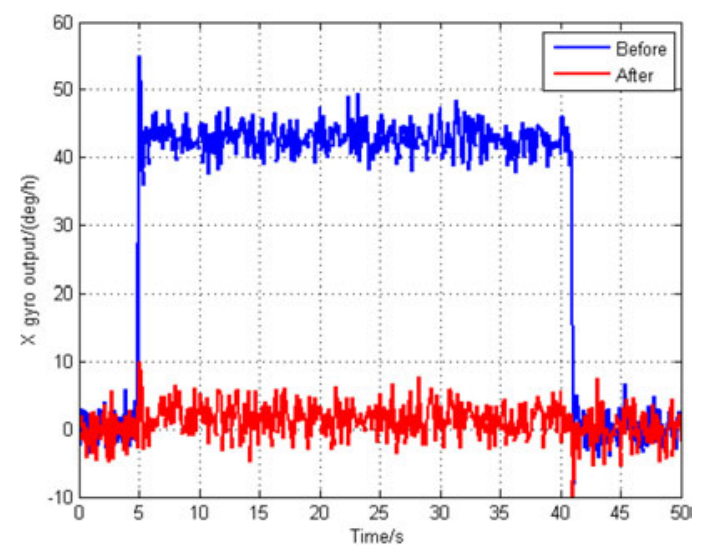

Figure 11. Gyroscope Outputs Before and After Non-orthogonality Calibration.

5.2. Calibration of Centripetal Acceleration Caused by Rotation. Since the centres of the three accelerometers are not exactly on the rotation axis, an additional acceleration caused by centripetal force will function as constant bias provided that the distance from accelerometers to rotation axis being fixed and the rotation rate being stable. This constant bias to one of gyroscopes described above cannot be modulated by rotation. It is compensated according to the following formula:

$$
\Delta a=D \cdot \Omega^{2}+\nabla
$$

where $D$ is the distance between accelerometer centres and rotation axis and $\nabla$ is the accelerometer constant bias. The calibration setting requirements are the same as the calibration of non-orthogonality of rotation axis and body coordinates, so these two compensating experiments can be conducted at the same time. As the IMU box is not a perfect cube, every distance must be measured. Record the accelerometer outputs and rotation rate, apply the Least Square method to them and estimate the distances $D_{x}, D_{y}, D_{z}$. The distances, which are listed in Table 3, can then be used to compensate for the centripetal force.

5.3. Calibration of the Synchronism of Gyroscope and Encoder. The gyroscopes and encoder are required to be synchronous to avoid errors of rotation compensation and errors of angular velocity in body coordinates. However, due to different electronic characteristics, the synchronism cannot be guaranteed without calibration. According to the user manuals, the gyroscope's group delay is 2 milliseconds while the encoder's signal latency is only $0 \cdot 250$ milliseconds.

That asynchronism leads to errors of rotation compensation for the gyroscope along $\mathrm{OZ}_{\mathrm{b}}$ is obvious, while the reason why asynchronism causes errors of angular velocity in body coordinates requires explanation. Assume that the asynchronous time 
Table 3. Calibration Results of Centripetal Acceleration Caused by Rotation.

\begin{tabular}{lccccccc}
\hline Items $(\mathrm{cm})$ & 1 & 2 & 3 & 4 & 5 & Average & Deviation \\
\hline$D_{x}$ & 3.45 & 3.86 & $3 \cdot 24$ & $3 \cdot 37$ & 3.64 & 3.512 & $0 \cdot 243$ \\
$D_{y}$ & 3.38 & 3.67 & 3.48 & 3.41 & 3.86 & 3.560 & $0 \cdot 202$ \\
$D_{z}$ & $1 \cdot 16$ & 1.58 & 1.65 & 1.21 & 1.75 & 1.470 & $0 \cdot 268$ \\
\hline
\end{tabular}

Table 4. Calibration Results of Synchronism of Gyroscope and Encoder.

\begin{tabular}{lccccccc}
\hline Items $(\mathrm{ms})$ & 12 & 2 & 3 & 4 & 5 & Average & Deviation \\
\hline$\Delta t$ & $4 \cdot 2$ & $4 \cdot 3$ & $4 \cdot 2$ & $4 \cdot 5$ & $4 \cdot 2$ & $4 \cdot 28$ & $0 \cdot 13$ \\
\hline
\end{tabular}

is $\Delta t$ and the vehicle is spinning around $\mathrm{OX}_{\mathrm{b}}$ at rate $\omega$, namely $\left[\omega_{x} \omega_{y} \omega_{z}\right]\left[\begin{array}{lll}\omega & 0 & 0\end{array}\right]$. If the IMU is rotated at rate $\Omega$, then gyroscope outputs along $\mathrm{OX}_{\mathrm{r}}$ and $\mathrm{OY}_{\mathrm{r}}$ are:

$$
\begin{aligned}
& \omega_{x}=\omega \cdot \cos (\Omega t) \\
& \omega_{y}=-\omega \cdot \sin (\Omega t)
\end{aligned}
$$

Considering the vehicle's spinning rate is much larger than the gyroscope constant biases and the Earth's rotation rate, they are ignored here. To get the angular velocity in body coordinate system, a transfer which involves the asynchronous time is made:

$$
\begin{aligned}
& \omega_{X}=\omega_{x} \cdot \cos [\Omega(t+\Delta t)]-\omega_{y} \cdot \sin [\Omega(t+\Delta t)]=\omega \cos (\Omega \Delta t) \approx \omega \\
& \omega_{Y}=\omega_{x} \cdot \sin [\Omega(t+\Delta t)]+\omega_{y} \cdot \cos [\Omega(t+\Delta t)]=\omega \sin (\Omega \Delta t) \approx \omega \Omega \Delta t
\end{aligned}
$$

The approximate condition is that $\Delta t$ is very short (several milliseconds). Thus the angular velocity errors in body coordinates are:

$$
\begin{aligned}
& \Delta \omega_{X}=0 \\
& \Delta \omega_{Y}=\Omega \cdot \omega \Delta t
\end{aligned}
$$

In conclusion, asynchronous time between gyroscope and encoder results in angular velocity errors along $\mathrm{OX}_{\mathrm{b}}\left(\mathrm{OY}_{\mathrm{b}}\right)$ if there is angular motion along $\mathrm{OY}_{\mathrm{b}}\left(\mathrm{OX}_{\mathrm{b}}\right)$. We can see through simple calculation that this error is extremely large and must be compensated.

Calibration experiments were conducted to determine the asynchronous time. RMSINS was mounted on a turntable, with $\mathrm{OX}_{\mathrm{b}}$ pointing to the rotation axis of the turntable at first. The IMU was powered up and then rotated at rate $\Omega=10^{\circ} / \mathrm{s}$ while the turntable was set to accelerate from zero to a certain rate, held at that rate for some time and then slowed down to zero at the same acceleration. Angular rate output along $\mathrm{OY}_{\mathrm{b}}$ was recorded. The transfer from rotation coordinates to body coordinates is done inside RMSINS, so the angular rate outputs are provided in the body coordinate system, as required for the calibration of asynchronous time. Results of five experiments are listed in Table 4. As the mathematical model required that 


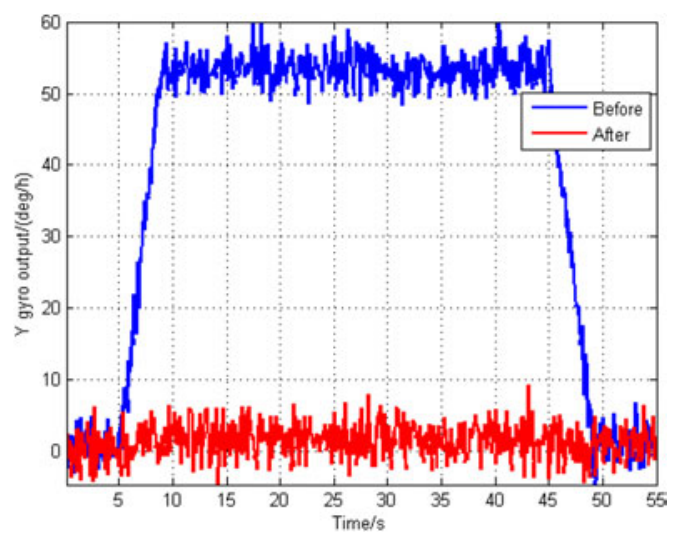

Figure 12. Gyroscope Outputs Before and After Synchronism Calibration.

the constant biases are small, online bias estimation was conducted and biases were removed before the calibration experiment began.

Figure 12 shows the cross-sensitivity angular rate error in conditions where the IMU's rotation rate $\Omega$ is $10 \%$ and turntable's maximum angular rate is $20 \%$ with an acceleration of $5 \% \mathrm{~s}^{2}$. It is apparent that the time delay between gyroscope and encoder will be a significant error, degrading the performance of RMSINS unless compensated.

\section{EXPERIMENTS}

6.1. Experiment Setup and Results. In order to verify the RM effects on navigation accuracy, experiments are conducted. The first experiment was a lengthy (1 hour) static attitude accuracy test under room temperature/pressure conditions. RMSINS was static on a platform performing the inertial navigation algorithm and attitudes were recorded. Results are shown in Figure 13. The aim of the experiment was to verify the RM effects on gyroscope constant biases, which play the key role in attitude errors. The long experiment duration will make the comparison more obvious.

The second experiment was a short (216 seconds) static navigation accuracy test under room temperature/pressure conditions. Comparisons in navigation accuracy before and after RM were made. RMSINS locked the motor and started inertial navigation just like conventional INS. Then the IMU started to spin after a set time and navigate again with the same initial conditions. During the whole procedure, the power of RMSINS was always on to eliminate the possibility that poor repeatability of switch on/off bias might weaken the verification. Results are shown in Figure 14. As the rotation axis is aligned with $\mathrm{OZ}_{\mathrm{b}}$, only results of pitch and roll whose accuracy is improved are shown for comparisons and explanations. Figure 14 (a), (b) and (c) show the accuracy comparisons in attitude, velocity and position respectively between after and before RM while (d) shows East velocity/position errors and roll error after RM. The simulation results are available in Section 2. The aim of this experiment was to verify the effects of the selected RM scheme on inertial navigation accuracy by comparing it with that of conventional INS. 

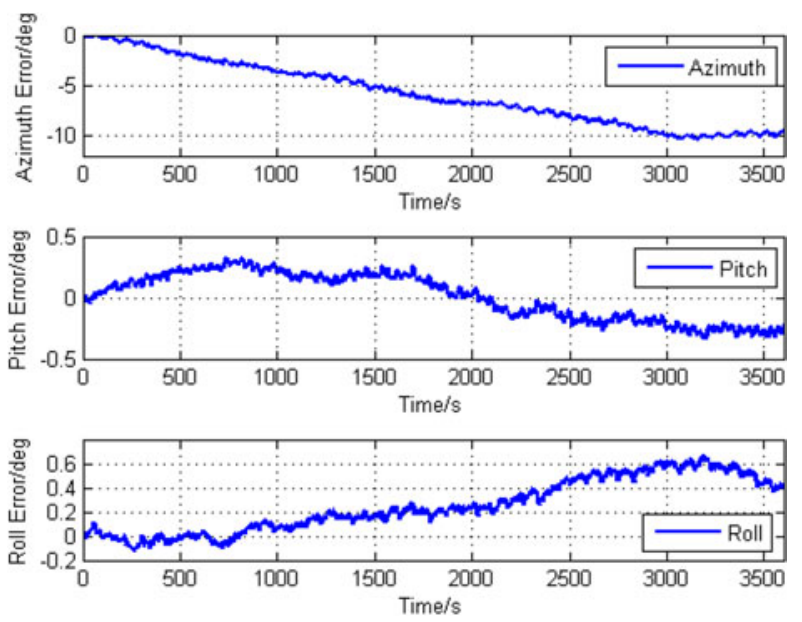

Figure 13. Results of 1 Hour Attitude Accuracy Test.
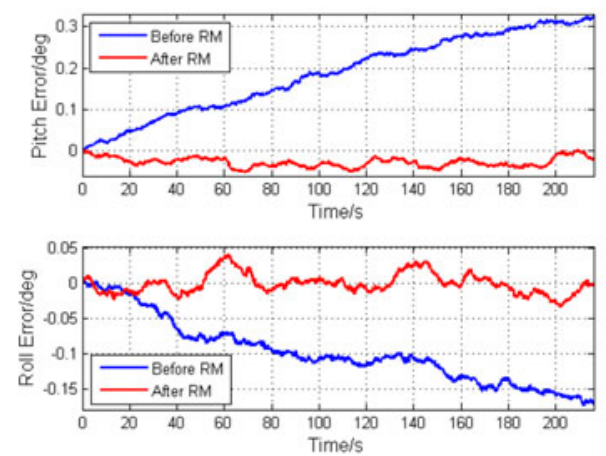

(a)Pitch/Roll Error Comparison
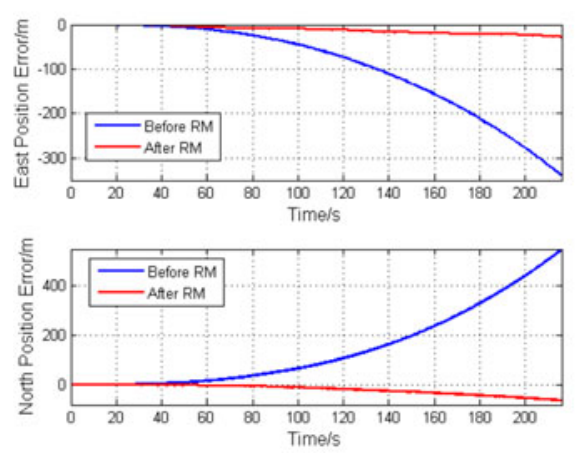

(c) East/North Position Error Comparison
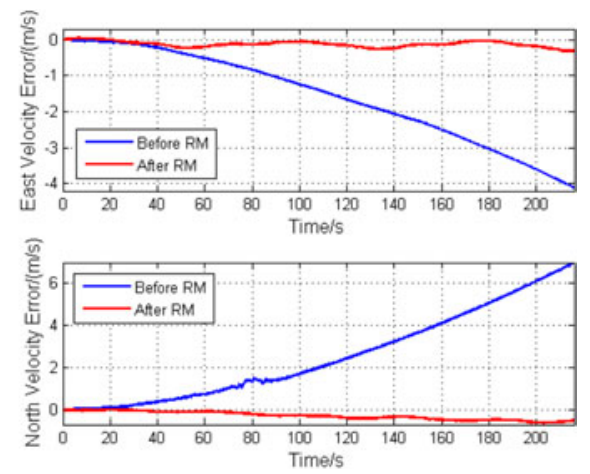

(b) East/North Velocity Error Comparison
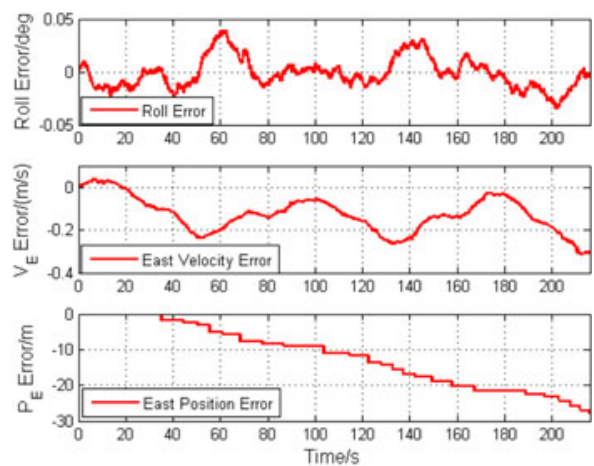

(d) East Channel Velocity/Position Error

Figure 14. Results of $216 \mathrm{~s}$ Inertial Navigation Experiment.

6.2. Explanations. The lengthy experiment proves that despite being calibrated, the gyroscope constant biases are still large due to the poor bias repeatability. As azimuth error indicates, the constant bias along $\mathrm{OZ}_{\mathrm{b}}$ is about $-10 \%$ and is the main 
Table 5. Navigation Accuracy Comparison Between After and Before RM.

\begin{tabular}{lcr}
\hline Maximum Error & After RM & Before RM \\
\hline Pitch $\left(^{\circ}\right)$ & -0.05 & $0 \cdot 33$ \\
Roll $\left({ }^{\circ}\right)$ & $0 \cdot 04$ & $-0 \cdot 18$ \\
East velocity $(\mathrm{m} / \mathrm{s})$ & $-0 \cdot 31$ & $-4 \cdot 12$ \\
North velocity(m/s) & -0.62 & $7 \cdot 02$ \\
East positon(m) & -28 & $-338 \cdot 5$ \\
North position(m) & -62 & $547 \cdot 8$ \\
\hline
\end{tabular}

source of orientation error. In contrast, the pitch and roll errors are much smaller because the constant biases along $\mathrm{OX}_{\mathrm{b}}$ and $\mathrm{OY}_{\mathrm{b}}$ have been modulated into zero-mean periodical values which generate much smaller orientation errors than the constants do. The maximum pitch and roll error is about $0.8 \%$ h, so after applying RM to INS, the attitude accuracy is improved by more than 10 times in 1 hour. It can also be seen that the pitch and roll errors are modulated by Schuler Oscillation, whose period is $84.4 \mathrm{~min}$, which proves that uncorrected constant bias is no longer the main error source after RM.

In the short navigation experiment, the improvement on navigation accuracy after introducing RM is significant as Figure 14 (a), (b), (c) and Table 5 indicate. The attitude accuracy is five times better while velocity/position accuracy is nearly ten times better. In Figure 14(a), the uncorrected constant biases are approximately $8 \% \mathrm{~h}$ and $-3.5 \%$ along $\mathrm{OX}_{\mathrm{r}}$ and $\mathrm{OY}_{\mathrm{r}}$ respectively, but after introducing $\mathrm{RM}$, the biases are almost zero. Due to better attitude accuracy, the velocity/position errors are greatly reduced, as shown in (b) and (c). Before RM, the velocity/position error caused by gyroscope constant biases can be estimated as follows:

$$
\begin{aligned}
& \Delta V_{E}=0 \cdot 5 \varepsilon_{y} g t^{2}=-3.88 \mathrm{~m} / \mathrm{s} \quad \Delta V_{N}=0.5 \varepsilon_{x} g t^{2}=8.86 \mathrm{~m} / \mathrm{s} \\
& \Delta P_{E}=\frac{1}{6} \varepsilon_{y} g t^{3}=-279 \mathrm{~m} \quad \Delta P_{N}=\frac{1}{6} \varepsilon_{x} g t^{3}=638 \mathrm{~m}
\end{aligned}
$$

It is clear that the errors caused by gyroscope constant biases compose nearly all of the velocity/position errors, which means accelerometer biases have much less influence, so the smaller gyroscope biases, the better the velocity/position accuracy. In Figure 14(d), the RM period that is 72 seconds can be seen. The difference in peak magnitudes of roll error is caused by the different gyroscope biases along $\mathrm{OX}_{\mathrm{r}}$ and $\mathrm{OY}_{\mathrm{r}}$. The velocity error varies in the period of 72 seconds, unlike that before RM, growing in proportion to the square of operation time.

In summary, by applying RM to MEMS-based SINS, constant biases and slowlychanging errors are turned into zero-mean periodical errors, so the inertial navigation accuracy is significantly improved.

7. CONCLUSION. Micro-electro-mechanical systems (MEMS) sensors have many advantages including low weight, small size, low cost and high reliability, but their errors are large and error repeatability is poor. Conventional calibrations that compensate bias, scale factors, non-orthogonalities and thermal effects are needed. However due to the poor repeatability of biases, the uncorrected biases are the 
dominant error sources for short time inertial navigation. Rotation Modulation (RM), which is a systematic auto-compensation method, was introduced to solve this problem. The modulation effects on navigation accuracy were discussed and expressed in analytical formulae. Different rotation schemes are compared and a suitable one for MEMS-based Strap-down Inertial Navigation System (SINS) was selected. Based on the chosen rotation scheme, a Rotation Modulation SINS based on MEMS sensors has been developed. Since rotation is involved, some more calibrations related to rotation are necessary, as well as the conventional calibrations. Experiment results verify that RM can reduce errors caused by constant biases, greatly improving the navigation accuracy of MEMS-based SINS. For short time (216 seconds) inertial navigation, the attitude and velocity/position accuracy are improved by five times and nearly ten times respectively. RM may extend the applications of MEMS-based SINS to some higher accuracy areas while still retaining most of their advantages.

\section{REFERENCES}

Aggarwal, P., Syed, Z., Niu, X., El-Sheimy, N. (2007). Thermal Calibration of Low Cost MEMS Sensors for Integrated Positioning, Navigation Systems. The Institute of Navigation National Technical Meeting, California, USA, January 22-24.

Aggarwal, P., Syed, Z., Niu, X., El-Sheimy, N. A. (2008). Standard Testing and Calibration Procedure for Low Cost MEMS Inertial Sensors and Units. The Journal of Navigation. 61, 323-336.

Brown, A. and Y. Lu (2004). Performance test results of an integrated GPS/MEMS inertial navigation package. Proceedings of ION GNSS Conference. Long Beach, CA.

Crain, Timothy P. II, Bishop, Robert H., Brady, Tye (2010). Shifting the inertial navigation paradigm with MEMS technology. Advances in the Astronautical Sciences, 137, 269-288.

El-Diasty, M., El-Rabbany, A., Pagiatakis, S. (2007). Temperature variation effects on stochastic characteristics for low-cost MEMS-based inertial sensor error. Measurement Science and Technology, 18, 3321-3328.

El-Diasty, M., El-Rabbany, A., Pagiatakis, S. (2006). Stochastic Characteristics of Temperature-Dependent MEMS-Based Inertial Sensor Error. The Institute of Navigation National Technical Meeting, Monterey, California, USA, January 18-20.

Giovanni, C. S., Levinson, E. (1981). Performance of a Ring Laser Strapdown Marine Gyrocompass. ION 7th Annual Meeting Proceedings, Annapolis, Maryland, USA.

Ishibashi, S., Aoki, T. (2006). The Method to Improve the Performance of an Inertial Navigation System using a Turntable. The Proceedings of The Sixteenth (2006) International Offshore and Polar Engineering Conference, San Francisco, USA.

Klass, J (1958). Gyro Drfit Cut by Bearing Technique. Aviation Week, 68, 79-81.

Lai, Ying-Chih, Jan, Shau-Shiun, Hsiao, Fei-Bin (2010). Development of a Low-Cost Attitude and Heading Reference System Using a Three-Axis Rotating Platform. Sensors, 10, 2472-2491.

Levinson, D. E., Majure, R. (1987). Accuracy enhancement techniques applied to the Marine Ring Laser Inertial Navigator (MARLIN). Journal of the Institute of Navigation, 34, 71-80.

$\mathrm{Li}$, An, Chang, Guo-Bin (2010). Improved precision of strapdown inertial navigation system brought by dual-axis continuous rotation of inertial measurement unit. Second International Asia Conference on Informatics in Control, Automation and Robotics, 1, 284-287.

Oliver, J. Woodman (2007). An Introduction to Inertial Navigation. Technical Report of University of Cambridge Bridge, 696.

Qian, Wei-Xing, Liu, Jian-Ye, Lai, Ji-Zhou, Zhang, Ling (2010). Initial alignment method for rotational strapdown inertial navigation system based on strong tracking filter. Journal of Chinese Inertial Technology, 18, 290-295.

Sun, Feng, Sun, Wei, Gao, Wei, Ben, Yueyang (2009). Research on the technology of rotational motion for FOG strapdown inertial navigation system. 2009 IEEE International Conference on Mechatronics and Automation, ChangChun, Jilin, CHN. 
Sun, Feng, Wang, Qiuying (2012). Researching on the compensation technology of rotating mechanism error in single-axis rotation strapdown inertial navigation system. 2012 IEEE International Conference on Mechatronics and Automation. Sichuan, CHN.

Titterton, D.H, Weston, J.L (2004). Strapdown Inertial Navigation Technology(2nd Edition). Peregrinus Ltd. Institution of Electrical Engineers, London.

Wang, Qi, Xu, Xiao-su (2007). Application of IMU rotation in FOG strapdown attitude and heading reference system . Journal of Chinese Inertial Technology, 15(3), 265-268.

Yang, Yong, Miao, Lingjuan, Shen, Jun (2005). Method of Improving the Navigation Accuracy of SINS by Continuous rotation. Journal of Beijing Institute of Technology. 14(1), 45-49. 